\title{
Urinary Tract Infection among Adults Seeking Medicare at Kiambu Level 5 Hospital, Kenya: Prevalence, Diversity, Antimicrobial Susceptibility Profiles and Possible Risk Factors
}

\author{
Fredrick Wanja ${ }^{1}$, Caroline Ngugi ${ }^{1}$, Eric Omwenga ${ }^{2}$, John Maina ${ }^{3}$, John Kiiru ${ }^{3}$ \\ ${ }^{1}$ Department of Medical Microbiology, Jomo Kenyatta University of Agriculture and Technology, Nairobi, Kenya \\ ${ }^{2}$ Department of Microbiology \& Parasitological, Kisii University, Kisii, Kenya \\ ${ }^{3}$ Centre of Microbiology Research, Kenya Medical Research Institute, Nairobi, Kenya \\ Email: *wanjakimunya@gmail.com
}

How to cite this paper: Wanja, F., Ngugi, C., Omwenga, E., Maina, J. and Kiiru, J. (2021) Urinary Tract Infection among Adults Seeking Medicare at Kiambu Level 5 Hospital, Kenya: Prevalence, Diversity, Antimicrobial Susceptibility Profiles and Possible Risk Factors. Advances in Microbiology, 11, 360-383.

https://doi.org/10.4236/aim.2021.118028

Received: July 1, 2021

Accepted: July 29, 2021

Published: August 2, 2021

Copyright $\odot 2021$ by author(s) and Scientific Research Publishing Inc. This work is licensed under the Creative Commons Attribution International License (CC BY 4.0).

http://creativecommons.org/licenses/by/4.0/

\begin{abstract}
Urinary tract infections are among the most prevalent extra-intestinal infections, with high prevalence globally. This cross-sectional study established prevalence of bacterial aetiology causing urinary tract infection (UTI) and their antimicrobial susceptibility profiles. A questionnaire was used to capture socio-demographic data and possible UTI risk factors among the 206 consented adults seeking medicare at Kiambu Level 5 Hospital. The collected midstream urine samples were subjected to dipstick analysis, microscopy and culture for UTI diagnosis. Results: The overall prevalence rate of UTIs was $27.6 \%$, with women's prevalence rate being significantly higher at $80.7 \%$ compared to men $19.2 \%$. Pregnant women had UTI prevalence at $34 \%$ which was higher than other sets of participants. Women who did not frequently change their underpants daily had a higher UTI cases at 34.8\%. Escherichia coli, Staphylococcus aureus and Klebsiella pneumoniae were the most prevalent bacterial pathogens at $38.5 \%, 21 \%$ and $19.3 \%$, respectively. Antimicrobial sensitivity analysis revealed high resistances towards Sulfamethoxazole and Ampicillin at range between $50 \%-85 \%$, suggesting that these drugs are no longer effective for UTI empirical treatment. The resistance patterns towards Cefotaxime, Cefepime and Ciprofloxacin were below 40\%. However, more resistance patterns at a range between $14 \%$ - $40 \%$ revealed towards Amoxicillin-clavulanic and Nitrofurantoin imply that these are drugs remain potent but there is the need to revise the current UTI management guidelines. In ad-
\end{abstract}


dition, to elude treatment failure, innovation of prophylactic measures is key to halt UTI contraction and offer support to pharmaceutical industries that have fewer new antibiotics in the pipeline.

\section{Keywords}

Urinary Tract Infection (UTI), Risk Factors, Antimicrobial Resistance and Antimicrobial Susceptibility Profile

\section{Introduction}

Urinary tract infections are still among the most prevalent extra-intestinal infections reported globally [1] [2]. Although these infections are not life-threatening, the high incidences significantly increase healthcare costs and negatively impact patients' quality of life [3]. UTIs complicate the clinical welfare of affected patients and consequently create substantial economic and social burdens [4] [5] [6]. If not promptly diagnosed, left unattended or mis-diagnosed, the infections further lead to severe health impacts like renal damage. However, sound knowledge of UTIs and associated risk factors allow timely intervention to quickly bring the disease under control [7].

The prevalence of UTIs among adults varies, but globally females are more prone, with an estimated prevalence of about $25 \%$ being reported [8]. This has been attributed to women having a shorter urethra that is closer to the anal opening than men [9]. Other predisposing factors making women more prone to urinary tract infections are; voiding, wiping technique, wearing of tight pants or undergarments and vaginal douching [10]. The prevalence of UTIs among males aged under 50 years is low compared to that of adult women of the same age bracket, who are thirty more times likely to develop a UTI [11]. Evidence from previously conducted studies across the globe also ascertains that UTI prevalence rates vary widely because of different factors like: poverty, literacy, sanitation infrastructures that come into place [12]. For instance, a study in the USA that involved over 10.8 million patients reported a prevalence rate of $16.7 \%$ [13]. Almost similar prevalence rates (11.2\%) were also reported in Asia [14] a clear indication that UTI prevalence rates differ across the globe. In Africa, related studies among adults have also revealed novel evidence that proves UTIs are an actual health burden [15] [16] [17] [18]. Recognizing these reported UTI prevalence trends and many more means that more research needs to be done to evaluate the prevalence, incidence and risk associated factors of urinary tract infections among adults.

The most prevalent etiological agents of urinary tract infections are bacteria [19]. This undeniable reality calls for more attentive investigations to understand and curb the menace. Among the bacteria known to cause UTIs, E. coli in particular has been documented to be the leading etiological agent [10] [20] [21]. 
Other Gram-negative bacteria that cause UTI include: Klebsiella pneumoniae, Proteus mirabilis and Pseudomonas aeruginosa [22]. Gram-positive bacteria that invade the urinary tract include: Staphylococcus, Streptococcus and Enterococcus species [23]. However, non-bacterial UTI etiological agents like Fungi, Viruses and Parasites have also been recorded in past related UTI studies [24]. Fundamentally, communities need to be enlightened on how to embrace all measures of UTI prevention and control. Furthermore, the larger spectrum of bacteriuria uropathogens and associated-risk factors remain under-investigated. Some of UTI risk factors reported in related studies include: Health conditions, Gender, Sexual activity, Pregnancy, Menopause, Age, Past history of UTI, Contraceptives and Social-economic status [12] [17].

Prompt UTI diagnosis and timely intervention is therefore very critical to halt pathogen establishment. Indeed, it is regrettable that most Kenyan health facilities that perform urine tests rely on rapid dipstick and direct wet microscopy urinalysis tests to diagnose UTI as they give immediate results and as such, urine culture is rarely done [25]. However, these tests have poor negative and positive predictive values to detect the presence of bacteriuria, especially in asymptomatic individuals [26]. Furthermore, the results of these tests are inadequate to inform the most probable drug of choice. Results of this study indicate that due to lack of microbial analysis, misdiagnosis may be a significant drive of UTI treatment failure. Again, considering that clinicians in most of these Kenyan facilities use empirical treatment to manage UTI. Prescription of antibiotics without relevant microbial findings, more so susceptibility patterns may be influencing the rising antimicrobial resistance. Culture and antimicrobial sensitivity tests are therefore the gold standard method of UTI diagnosis and as such should be embraced to inform management. However, innovation of new therapeutic breakthroughs and updating of the current UTI management policies is indispensable. This study's main objective was therefore to investigate and determine the prevalence of bacterial urinary tract infection, antimicrobial susceptibility profiles, and UTI associated risk factors among adults attending Kiambu level 5 Hospital.

\section{Materials and Methods}

\subsection{Study Design, Site and Ethical Approval}

This cross-sectional study was done in Kiambu level 5 Hospital, Kenya. Study ethical approval was sought from the Kenyatta National Hospital-University of Nairobi ethical research committee (Reference no: KNH-ERC/A/470; Supplementary data S1) and National Commission for Science, Technology, and Innovation (NACOSTI) (Reference no: 619853; Supplementary data S2).

\subsection{Sample Size and Sample Collection}

A purposive sampling technique was applied to recruit patients until the study sample size of 206 was achieved. The sample size was determined using the for- 
mula by Lwanga and Lemeshow's 1991 using a UTI prevalence rate of $13.3 \%$ reported by Kabugo et al. 2016 [18].

$$
N=1.96^{2} \times P Q / D^{2}
$$

$N=$ Desired minimal sample size.

$Z=$ Standard normal deviation (1.96 from the tailed normal table).

$P=$ Prevalence of the condition under study.

$Q=1-P$.

$D=$ Precision required for the study at $95 \%$ confidence level (0.05).

Calculation of the sample size

$$
\begin{gathered}
P=13.3 Q=1-0.133 D=0.05 N=1.96^{2} \times P Q / D^{2} \\
N=1.96^{2} \times\left(0.133 \times 0.867 / 0.05^{2}\right) \\
N=177
\end{gathered}
$$

However, two hundred and six (206) midstream urine samples were collected which are above the 177 required (206; women-77.6\% \& men-22.3\%).

Using well-structured questionnaires, participants' social demographic, clinical and lifestyle information was collected after giving consent to participate. Participants were given explicit instruction on the proper approach in collecting a midstream sample in a sterile collection tube [1] [27].

\subsection{Urine Sample Analysis}

\subsubsection{Macroscopic, Dipstick and Microscopic Urine Analysis}

All 206 participant's urine samples were subjected to macroscopy observation to test various pathological parameters. Dipstick analysis using the 10-parameter chemical reagent urine strip and microscopic examination was performed as previously documented [15] [18]. Briefly, $10 \mathrm{ml}$ of the urine sample was centrifuged at 2000 - 3000 rounds per minute (rpm) for 5 minutes. The supernatant after centrifugation was poured and a drop of the deposit placed on a glass microscope slide, covered with a cover slip and examined using a compound microscope (OLYMPUS CX23, JAPAN) under 10× objective len. This was followed by examination under $40 \times$ objectives. This was to determine the presence of pus cells, white blood cells, bacteria or yeast, casts, crystals and red blood cells in urine [28]. Any bacteria presence detected (1 - 4 per high power field) was considered as bacteriuria. In contrast, the presence of pus cells/leucocytes $\geq 10$ in a single high-power field (HPF) was treated as pyuria case [15].

\subsubsection{Bacterial Isolates Identification}

\section{1) Urine Culture}

Urine culturing was done using previously established protocols [27]. Participant's $5 \mu \mathrm{l}$ urine samples that met the urine microscopy threshold of pus cells/leucocytes $\geq 10$ in a single high-power field were cultured using surface streaking technique on Cysteine Lactose Electrolyte Deficient (CLED) and MacConkey agar plates (Oxoid, UK). The cultures were then aerobically incubated at $37^{\circ} \mathrm{C}$ for 18 to 24 hours as done before [29]. Later the isolated cultures 
were subsequently sub-cultured on Mueller Hinton agar (Oxoid, UK) to obtain pure cultures that were used for Gram stain reaction and a series of biochemical test recommended for enteric and Staphylococcus species identification. Both the significant $\left(\geq 10^{5} \mathrm{CFU} / \mathrm{mL}\right)$ and the non-significant $\left(\leq 10^{5} \mathrm{CFU} / \mathrm{mL}\right)$ bacterial growth were concurrently investigated, and their profiles analysed. $S$. aureus (ATCC-25923), E. coli (ATCC-25922) and Pseudomonas aeruginosa (ATCC-27853) were used as quality control strains (QC). All protocols were done independently of each other as per previously used techniques [22] [27] [30].

\section{2) Colony morphology}

Though bacterial colony morphologies differ on various culture media, this study colonial morphology on CLED culture plates were determined based on; Pigmentation-color of the colony, Size-pinpoint, small, moderate, or large, Form-circular, irregular, or rhizoid, Elevation-flat, raised, convex, or umbonate and Margin-entire, lobate, undulate, serrate or, filamentous. S. aureus (ATCC-25923), E. coli (ATCC-25922) and Pseudomonas aeruginosa (ATCC-27853) were used as quality control strains (QC) (Supplementary data S3a, S3b, S3c). All protocols were done as per previously used techniques [22] [27] [30].

\section{3). Colony forming units}

Bacterial colonies on the primary CLED plate were counted to determine the yielded colony-forming units. Bacterial growth yielding colony-forming units of $\geq 100,000 \mathrm{CFU} / \mathrm{ml}\left(10^{5}\right)$ and above were assumed to have met the threshold/cut off point of UTI positive and were considered significant. Those yielding lower growth below $\leq 100,000 \mathrm{CFU} / \mathrm{ml}$ were deemed as non-significant growth/contaminants.

\section{4) Gram staining}

Gram staining was conducted prior to biochemical tests to classify and provide preliminary results of the isolates, this identified the isolates into either Gram-positive or negative by use of established protocol [19]. The prepared smears were left to air dry after staining awaiting observation under oil immersion (100x) using a compound microscope. S. aureus (ATCC-25923), E. coli (ATCC-25922) and Pseudomonas aeruginosa (ATCC-27853) were used as quality control strains (QC). Procedure was done as per previously used techniques [22] [27] [30].

\section{5) Biochemical tests}

Biochemical tests were conducted as per previously protocol to profile the isolates according to standard bacterial classification to the species level [31]. The following biochemical tests were performed; Sulfur Indole Motility, Urea utilization, Citrate utilization, Triple sugar iron, Catalase, and Coagulase test [32]. Standard reference strains: $S$. aureus (ATCC-25923) and E. coli (ATCC-25922) were included to validate the study findings. All these tests were carried out independently of each other.

\subsection{Bioassays}

\section{Susceptibility Testing}

Antimicrobial susceptibility testing of isolates was performed according to the 
Kirby-Bauer disc diffusion method [33] on Mueller Hinton agar (Oxoid, United Kingdom). Test drugs were antimicrobials routinely used in management of both Gram-positive and Gram-negative bacteria infections. They included Ampicillin (AMP $10 \mu \mathrm{g}$ ), Amoxicillin-clavulanic acid (AMC, 20/10 $\mu \mathrm{g}$ ), Ceftriaxone (CRO, $30 \mu \mathrm{g}$ ), Nitrofurantoin (NIT, $300 \mu \mathrm{g}$ ), Gentamin (GEN, $10 \mu \mathrm{g}$ ), Cefoxitin (FOX, $30 \mu \mathrm{g}$ ), Erythromycin (ERY, $15 \mu \mathrm{g}$ ), Ciprofloxacin (CIP, $5 \mu \mathrm{g}$ ), Cefepime (FEP, $30 \mu \mathrm{g}$ ), Rifampicin (RIF, $15 \mu \mathrm{g}$ ), Nalidixic acid (NAL, $30 \mu \mathrm{g}$ ), Ceftazidime (CAZ, $30 \mu \mathrm{g}$ ), Cefotaxime (CTX, $30 \mu \mathrm{g}$ ), Sulfamethoxazole (SMX, $23.75 \mu \mathrm{g}$ ), Trimethoprim (TMP, $1.25 \mu \mathrm{g}$ ), Chloramphenicol (CHL, $30 \mu \mathrm{g}$ ), Tetracycline (TCY, $30 \mu \mathrm{g}$ ), Linezolid (LNZ, $30 \mu \mathrm{g}$ ), Vancomycin (VAN, $30 \mu \mathrm{g}$ ), Aztreonam (ATM, $30 \mu \mathrm{g})$. Comparable resistance patterns of the Gram-positive and Gram-negative isolates were done to determine the sensitivity trends against most of the study test antibiotics [34]. A double-disk synergy test to detect the likelihood of isolates carriage of ESBLs was performed using disks of $3^{\text {rd }}$ generation Cephalosporins and Cephalosporin-inhibitor (Clavulanic acid) antimicrobial disk [35] [36].

All tests were done independently of each other. Standard reference Staphylococcus aureus (ATCC-25923), Escherichia coli (ATCC-25922) and Pseudomonas aeruginosa (ATCC-27853) were used as controls to ensure the potency performance of the antibiotic discs and the quality of the media was assured.

\subsection{Data Analysis}

The study UTI prevalence rate was determined by dividing UTI positive cases with the total number of urine cultures done and expressed as a percentage. Antimicrobial inhibition zones were interpreted using the clinical and laboratory standard institute (CLSI) standards and expressed as either sensitive(S), intermediate (I) or resistance (R) as documented before [35]. The study findings were later entered into excels spreadsheets for analysis using statistical package for social sciences (SPSS) version 15. Chi-square test analysis was applied to determine the association between socio-demographic factors and probable UTI risk associated factors. Binary logistic regression analysis was carried out to generate the adjusted odds ratio with a 95\% confidence interval. An alpha of less than 0.05 (P $<0.05)$ was considered statistically significant.

\section{Results}

\subsection{Study Population}

The study involved adult patients seeking medicare at Kiambu level 5 Hospital; outpatient 174 (84.5\%) and inpatient 32 (15.5\%). The overall age mean among study participants was 31.8 years.

\subsection{Bacterial Identification}

\section{Biochemical Tests Results}

According to the study biochemical finding a variety of bacterial genera was re- 
sponsible for UTI among the study population (Table 1). The commonest isolated bacteria was E. coli at (38.5\%), followed by Staphylococcus aureus (21\%) and Klebsiella pneumonia (19.3\%) respectively. Other bacterial etiological agents isolated included: Proteus mirabilis (10.5\%), Staphylococcus saprophyticus (8.7\%) and Enterococcus faecalis (1.7\%). Data among the non-significant growths revealed Staphylococcus aureus and E. coli as the most prevalent bacteria accounting for $24.1 \%$ each. Klebsiella pneumoniae and Enterococcus faecalis followed at $17.2 \%$, and at $13.7 \%$ respectively. While Staphylococcus saprophyticus and Proteus mirabilis were the least isolated bacteria at $10.3 \%$ each.

\subsection{Prevalence of Urinary Tract Infection}

This study overall UTI prevalence was $27.6 \%$. UTI prevalence rate based on department category revealed most cases from the outpatient department at $73.6 \%$ (42) to inpatient $26.3 \%$ (15). In addition, female participants in the out-patient department had $88.3 \%$ UTI positive cases to men $11.6 \%$. However, a relatively high UTI prevalence rate was revealed among the female cohort attending antenatal clinic at $34 \%$ when matched to the rest of the outpatient sets of participants. In the inpatient department, a UTI prevalence rate of $66.6 \%(10 / 15)$ was revealed among women compared to men's 33.3\% (5/15). Even so, UTIs prevalence rates based on lifestyle factors and socio-demographic characteristics also seemed to heighten among the different sets of participants. The noted UTI prevalence rate amongst women participants of $80.7 \%$ was high than that amongst men of $19.2 \%$.

Table 1. Biochemical test findings.

\begin{tabular}{|c|c|c|c|c|c|c|c|c|c|}
\hline \multirow{3}{*}{$\begin{array}{c}\text { Test } \\
\text { Coagulase }\end{array}$} & & \multicolumn{8}{|c|}{ Gram staining $N=86$} \\
\hline & & \multicolumn{3}{|c|}{$\begin{array}{c}\text { Gram negative } \\
(\text { rods/bacilli) } \mathrm{n}=56(61.5 \%)\end{array}$} & \multirow{2}{*}{$\begin{array}{c}\text { E. coli } \\
\text { ATCC-25922 } \\
-\end{array}$} & \multicolumn{3}{|c|}{$\begin{array}{l}\text { Gram positive (cocci/sphere) } \\
\qquad \mathrm{n}=35(38.4 \%)\end{array}$} & \multirow{2}{*}{$\begin{array}{c}\text { S. aureus } \\
\text { ATCC-2 } \\
5923 \\
+\end{array}$} \\
\hline & & - & - & - & & + & - & - & \\
\hline Catalase & & - & - & + & - & + & - & + & + \\
\hline Indole & & + & + & - & + & - & - & - & - \\
\hline Citrate & & + & - & + & - & - & - & - & - \\
\hline Urease & & + & - & + & - & + & - & - & + \\
\hline $\begin{array}{c}\text { Triple Sugar Iron } \\
\text { Test }\end{array}$ & & + & + & + & + & - & - & - & - \\
\hline Sulfur Test & & + & + & - & + & - & - & - & - \\
\hline \multirow[t]{3}{*}{ Motility test } & & + & + & - & + & - & - & - & - \\
\hline & $\begin{array}{l}\text { Probable } \\
\text { Pathogen }\end{array}$ & $\begin{array}{l}\text { Proteus } \\
\text { mirabilis }\end{array}$ & E. coli & K. Pneumonia & & S. aureus & E. faecalis & S. Saprophyticus & \\
\hline & Number (\%) & $9(10.5 \%)$ & $29(33.7 \%)$ & $16(18.6 \%)$ & & $19(22.1 \%)$ & $5(5.8 \%)$ & $8(9.3 \%)$ & \\
\hline
\end{tabular}

Key: positive test result (+), negative test result (-). E. coli ATCC-25922 used as Gram-negative control, S. aureus ATCC-25923 used as Gram-positive control. 
Possible Lifestyle Factors and Socio-Demographic Characteristics

1) Urinary tract infections in relation to patients age

Several possible lifestyle factors and socio-demographic characteristics were scrutinized to determine their association with UTI contraction. UTI occurrence among the study population based on age revealed participants between the ages of 18 to 50 years had a prevalence of $24.4 \%(43 / 176)$. This was lower when matched to that among participants within the age range of 21 - 30 years of $28.2 \%$. However, those aged between the ages of 41 - 50 had the highest UTI prevalence rate of $42.9 \%$ compared to the rest of the age cohorts (Table 2).

Table 2. Urinary tract infections in relation to patients' age

\begin{tabular}{|c|c|c|c|}
\hline Characteristics & No. (\%) obtained & With UTI & Without UTI \\
\hline \multicolumn{4}{|l|}{ Age in years } \\
\hline $18-20$ & $19(9.2 \%)$ & $6(31.6 \%)$ & $13(68.4 \%)$ \\
\hline $21-30$ & $96(46.6 \%)$ & $25(26 \%)$ & $71(73.9 \%)$ \\
\hline $31-40$ & $44(21.4 \%)$ & $11(25.0 \%)$ & $33(75.0 \%)$ \\
\hline $41-50$ & $14(6.8 \%)$ & $6(42.9 \%)$ & $8(57.1 \%)$ \\
\hline$>50$ & $33(16 \%)$ & $9(27.3 \%)$ & $24(72.7 \%)$ \\
\hline$>30$ years & & 31 & 84 \\
\hline$\leq 30$ years & & 26 & 65 \\
\hline Odds of having UTI $>30$ & & 0.4 & \\
\hline Odds of having UTI $\leq 30$ & & 0.42 & \\
\hline Odds ratio & & 0.953 & \\
\hline $\mathrm{p}$-value & & 0.876 & \\
\hline Confidence interval & & {$[0.52-1.75]$} & \\
\hline \multicolumn{4}{|l|}{ Marital status } \\
\hline Single & $89(43.2 \%)$ & $19(21.3 \%)$ & $70(78.7 \%)$ \\
\hline Married & $97(47.1 \%)$ & $30(30.9 \%)$ & $67(69.1 \%)$ \\
\hline Divorced & $6(2.9 \%)$ & $3(50.0 \%)$ & $3(50.0 \%)$ \\
\hline Widowed & $14(6.8 \%)$ & $8(57.1 \%)$ & $6(42.9 \%)$ \\
\hline Married & & 30 & 67 \\
\hline Single $\backslash$ Divorced $\backslash$ Widowed & & 30 & 79 \\
\hline Odds of having UTI among those married & & 0.448 & \\
\hline $\begin{array}{l}\text { Odds of having UTI among } \\
\text { those singleldivorced } \mid \text { widowed }\end{array}$ & & 0.38 & \\
\hline Odds ratio & & 1.179 & \\
\hline $\mathrm{p}$-value & & 0.592 & \\
\hline Confidence interval & & {$[0.646-2.152]$} & \\
\hline
\end{tabular}

Key: Age in years; $\leq 18-20,21-30,31-40,41-50,>50$. Marital status as; single, married, divorced, widowed. 
2) Urinary tract infections in relation to sexual activities

Sexual activities among the study participants were scrutinized to determine any relation with UTI occurrence. A urinary tract infection prevalence rate of $30.4 \%$ was revealed among participants with multiple sexual partners, higher than that of $27.6 \%$ among the cohort who had only one partner. Participants who had sexual intercourse more than twice weekly had UTI prevalence rate of $32.2 \%$, almost double that of $16.2 \%$ reported among participants who had sexual activities once weekly. Nevertheless, participants with no partners at all had the least urinary tract prevalence rate at $21.1 \%$ (Table 3 ).

Table 3. Urinary tract infections in relation to sexual activities.

\begin{tabular}{|c|c|c|c|}
\hline Characteristics & No. (\%) obtained & With UTI & Without UTI \\
\hline \multicolumn{4}{|l|}{ Frequency of sexual activity } \\
\hline Once & $37(17.9 \%)$ & $6(16.2 \%)$ & $31(83.7 \%)$ \\
\hline Twice & $5(2.4 \%)$ & $0(0.0 \%)$ & $5(100 \%)$ \\
\hline$>2$ & $121(58.7 \%)$ & $39(32.2 \%)$ & $82(67.8 \%)$ \\
\hline None & $43(20.8 \%)$ & $12(27.9 \%)$ & $31(72.1 \%)$ \\
\hline Once or more & & 48 & 115 \\
\hline None & & 12 & 31 \\
\hline $\begin{array}{l}\text { Odds of having UTI among those } \\
\text { having sexual intercourse } \geq \text { once per week }\end{array}$ & $\square$ & 0.417 & \\
\hline $\begin{array}{c}\text { Odds of having UTI among those } \\
\text { having no sexual intercourse per week }\end{array}$ & & 0.387 & \\
\hline Odds ratio & & 1.078 & \\
\hline p-value & & 0.844 & \\
\hline Confidence interval & & {$[0.511-2.275]$} & \\
\hline \multicolumn{4}{|l|}{ No. of sexual partners } \\
\hline One & $141(68.4 \%)$ & $39(27.6 \%)$ & $102(72.3 \%)$ \\
\hline Multiple & $46(22.3 \%)$ & $14(30.4 \%)$ & $32(69.6 \%)$ \\
\hline None & $19(9.2 \%)$ & $4(21.1 \%)$ & $15(78.9 \%)$ \\
\hline One or more & & 56 & 131 \\
\hline None & & 4 & 15 \\
\hline $\begin{array}{l}\text { Odds of having UTI among those } \\
\text { having more than one sexual partner }\end{array}$ & & 0.427 & \\
\hline $\begin{array}{l}\text { Odds of having UTI among those } \\
\text { with no sexual partner }\end{array}$ & & 0.267 & \\
\hline Odds ratio & & 1.603 & \\
\hline p-value & & 0.417 & \\
\hline Confidence interval & & {$[0.509-5.045]$} & \\
\hline
\end{tabular}

Key: Frequency of sexual activity on daily basis presented as; either once, twice, $>2$ and none. No. of sexual partners presented as; one, multiple or none. 


\section{3) Urinary tract infections in relation to personal hygiene}

Finding on poor personal hygiene had some high UTI prevalence rates revealed. For instance, the prevalence rate of UTI in participants who changed their undergarments once daily was $29.4 \%$, lower than that of $34 \%$ reported in the cohort that did change undergarments twice daily. But almost similar UTI prevalence finding was reported among participants who changed their sanitary towels twice daily during menses of $28.9 \%$. It was only among women who changed their sanitary towels once daily during menses that a high UTI prevalence rate of $76.6 \%$ was noted (Table 4 ).

\section{4) Urinary tract infections in relation to other demographic factors}

Urinary tract infection occurrences based on education background revealed a prevalence rate of $43.5 \%$ among the set of participants with primary education background. That urinary tract infection prevalence rate is high if matched to that of $28.6 \%$ and $24.6 \%$ among participants' with secondary and tertiary education backgrounds. The UTI prevalence rate among those with high blood pressure recording of $36.1 \%$ was also high than that of $25.1 \%$ amongst those with low blood pressure (Table 5).

\subsection{Antimicrobial Susceptibility Profiles}

\subsubsection{Antimicrobial Susceptibility Profiles of Gram-Positives}

Comparative analysis of Gram-positive antimicrobial resistance phenotypes from significant growths and non-significant growths unveiled resemblance. Staphylococcus aureus displayed resistance against Nitrofurantoin and Ceftriaxone at $18 \%$ and $29 \%$ respectively. The resistance of $S$. saprophyticus isolates against Ceftriaxone, Erythromycin and Ampicillin was at a range of $20 \%$ to $60 \%$. However, considering resistance profile findings of the study Gram-positive isolates against Nalidixic acid, Sulfamethoxazole and Erythromycin at a range of $60 \%$ to $100 \%$, then these drugs can no longer be used as drugs of choice for empirical treatment (Table 6).

\subsubsection{Antimicrobial Susceptibility Profiles of Gram-Negatives}

Gram-negatives isolates were the most prevalent, with Escherichia coli being the most frequent bacteria isolate. Although most Gram-negative isolates displayed resistance patterns, Escherichia coli isolates were exemplary, exhibiting antimicrobial and multidrug resistance trends. Most Gram-negative isolates had high resistances towards Sulfamethoxazole, Nalidixic acid and Ampicillin of 64\%, $50 \%$ and $27 \%$ respectively. However, reassuring observation was that most of these Gram-negatives isolates still exhibited low resistance to Cefepime 9\%, Cefoxitime $10 \%$ and Nitrofurantoin $14 \%$ suggesting that these drugs are still suitable for treatment. About $40 \%$ of Klebsiella pneumoniae and Proteus mirabilis isolates showed resistance patterns against Ampicillin at a range of $17 \%$ to $67 \%$, Sulfurmethoxazole $40 \%$ to $80 \%$ and Nalidixic acid $60 \%$ to $100 \%$ respectively (Table 7). 
Table 4. Urinary tract infections in relation to personal hygiene.

\begin{tabular}{|c|c|c|c|}
\hline Characteristics & No. (\%) obtained & With UTI & Without UTI \\
\hline \multicolumn{4}{|l|}{ Frequency of changing undergarments } \\
\hline Once & $112(54.3 \%)$ & $33(29.4 \%)$ & $79(70.5 \%)$ \\
\hline Twice & $53(25.7 \%)$ & $18(34.0 \%)$ & $35(66.0 \%)$ \\
\hline$>2$ & $41(19.9 \%)$ & $3(33.3 \%)$ & $38(86.4 \%)$ \\
\hline Odds of having UTI among those changing undergarment once & & 0.371 & \\
\hline Odds of having UTI among those changing undergarment more than once & & 0.512 & \\
\hline Odds ratio & & 0.725 & \\
\hline p-value & & 0.327 & \\
\hline Confidence interval & & {$[0.382,1.378]$} & \\
\hline \multicolumn{4}{|l|}{ Undergarment material } \\
\hline Other Fabrics & $116(56.3 \%)$ & $36(26.7 \%)$ & $80(73.3 \%)$ \\
\hline Cotton & $90(43.6 \%)$ & $24(31.0 \%)$ & $66(69.0 \%)$ \\
\hline Odds of having UTI among those whose undergarment material is not cotton & & 0.45 & \\
\hline Odds of having UTI among those whose undergarment material is cotton & & 0.364 & \\
\hline Odds ratio & & 1.238 & \\
\hline p-value & & 0.495 & \\
\hline Confidence interval & & {$[0.672-2.279]$} & \\
\hline \multicolumn{4}{|l|}{ How frequently do you take a shower daily } \\
\hline Once & $139(67.4 \%)$ & $35(25.1 \%)$ & $104(74.8 \%)$ \\
\hline Twice & $67(32.5 \%)$ & $22(32.8 \%)$ & $45(67.2 \%)$ \\
\hline$>2$ & $0(0 \%)$ & $0(0.0 \%)$ & $0(0.0 \%)$ \\
\hline Odds of having UTI among those taking shower once daily & & 0.376 & \\
\hline Odds of having UTI among those taking shower more than once daily & & 0.489 & \\
\hline Odds ratio & & 0.77 & \\
\hline p-value & & 0.417 & \\
\hline Confidence interval & & {$[0.409-1.447]$} & \\
\hline \multicolumn{4}{|l|}{ UTI in relation to frequent to changing sanitary towel during your menses (females) } \\
\hline Once & $30(14.5 \%)$ & $23(76.6 \%)$ & $7(23.3 \%)$ \\
\hline Twice & $83(40.2 \%)$ & $24(28.9 \%)$ & $59(71.1 \%)$ \\
\hline$>2$ & $47(22.8 \%)$ & $10(21.3 \%)$ & 37 (78.7\%) \\
\hline None (women on menopause and men) & $46(22.3 \%)$ & $0(0.0 \%)$ & $46(100.0 \%)$ \\
\hline Odds of having UTI among those changing sanitary towel once or less daily & & 0.52 & \\
\hline Odds of having UTI among those changing sanitary towel more than once daily & & 0.354 & \\
\hline Odds ratio & & 1.468 & \\
\hline p-value & & 0.221 & \\
\hline Confidence interval & & {$[0.794-2.714]$} & \\
\hline
\end{tabular}

Key: Frequency of changing undergarments, frequency of taking a shower daily, frequency of changing sanitary towel during your menses presented as; either once, twice or $>2$. Undergarment material presented as; either cotton or other fabrics. 
Table 5. Urinary tract infections in relation to other demographic factors.

\begin{tabular}{|c|c|c|c|}
\hline Characteristics & No. (\%) obtained & With UTI & Without UTI \\
\hline \multicolumn{4}{|l|}{ Occupation } \\
\hline Employed & $78(37.9 \%)$ & $29(37.2 \%)$ & $49(62.8 \%)$ \\
\hline Unemployed & $128(62.1 \%)$ & $31(24.2 \%)$ & $97(75.8 \%)$ \\
\hline Odds of having UTI among those employed & & 0.592 & \\
\hline Odds of having UTI among those unemployed & & 0.32 & \\
\hline Odds ratio & & 1.852 & \\
\hline p-value & & 0.048 & \\
\hline Confidence interval & & {$[1,3.41]$} & \\
\hline \multicolumn{4}{|l|}{ Education } \\
\hline Primary & $23(11.2 \%)$ & $10(43.5 \%)$ & $13(56.5 \%)$ \\
\hline Secondary & $98(47.6 \%)$ & $28(28.6 \%)$ & $70(71.4 \%)$ \\
\hline Tertiary & $69(33.5 \%)$ & $17(24.6 \%)$ & $52(75.4 \%)$ \\
\hline None & $16(7.8 \%)$ & $5(31.3 \%)$ & $11(68.8 \%)$ \\
\hline Odds of having UTI among those with primary level and below & & 0.625 & \\
\hline Odds of having UTI among those with secondary or tertiary level & & 0.369 & \\
\hline Odds ratio & & 1.694 & \\
\hline p-value & & 0.155 & \\
\hline Confidence interval & & {$[0.817,3.516]$} & \\
\hline \multicolumn{4}{|l|}{ Blood Pressure } \\
\hline H-High & $36(17.5 \%)$ & $13(36.1 \%)$ & $23(63.9 \%)$ \\
\hline L-Low & $8(3.9 \%)$ & $2(25.0 \%)$ & $6(75.0 \%)$ \\
\hline Odds of having UTI among those with abnormal blood pressure & & 0.517 & \\
\hline Odds of having UTI among those with normal blood pressure & & 0.385 & \\
\hline Odds ratio & & 1.345 & \\
\hline p-value & & 0.415 & \\
\hline Confidence interval & & {$[0.66,2.74]$} & \\
\hline
\end{tabular}

Key: Occupation presented as; either employed or unemployed. Education presented as; primary, secondary, tertiary or none. Blood pressure readings presented as; normal, high or low.

Table 6. Resistance profiles of Gram-negative isolated bacteria pathogens.

\begin{tabular}{|c|c|c|c|c|c|c|c|c|c|c|c|c|c|c|c|c|c|}
\hline Organism & Comment & $n$ & AMC & AMP & CAZ & $\mathrm{CHL}$ & CIP & CRO & CTX & FEP & FOX & GEN & NAL & NIT & SMX & TCY & TMP \\
\hline \multirow{2}{*}{ E. coli } & UTI & 22 & 24 & 27 & 9 & 5 & 0 & 18 & 18 & 9 & 10 & 23 & 50 & 14 & 64 & 46 & 67 \\
\hline & Contaminants & 7 & 14 & 43 & 0 & 0 & 0 & 14 & 14 & 0 & 0 & 0 & 57 & 14 & 57 & 71 & 71 \\
\hline \multirow{2}{*}{$\begin{array}{l}\text { Proteus } \\
\text { spp. }\end{array}$} & UTI & 6 & 0 & 17 & 0 & 0 & 0 & 0 & 17 & 17 & 0 & 17 & 33 & 17 & 83 & 0 & 67 \\
\hline & Contaminants & 3 & 33 & 67 & 0 & 67 & 0 & 33 & 33 & 0 & 33 & 0 & 100 & 33 & 100 & 67 & 67 \\
\hline \multirow{2}{*}{$\begin{array}{l}\text { Klebsiella } \\
\text { spp. }\end{array}$} & UTI & 11 & 18 & 27 & 27 & 18 & 0 & 27 & 27 & 18 & 18 & 27 & 36 & 27 & 9 & 9 & 18 \\
\hline & Contaminants & 5 & 20 & 20 & 0 & 20 & 0 & 0 & 0 & 0 & 0 & 20 & 60 & 20 & 40 & 40 & 0 \\
\hline
\end{tabular}




\subsubsection{Resistance Profiles of Isolated Bacterial Pathogens Based on Department Category}

Most of the bacteria isolates were obtained from the outpatient department $42 / 174$ (73.7\%) to inpatient 15/32 (26.3\%). Resistance patterns based on department category revealed a 33\% resistance trend by E.coli isolates from the inpatient against Ampicillin, Amoxicillin-clavulanic acid, Ciprofloxacin and Cefepime. In addition, Enterococcus species and S.saprophyticus isolates obtained from the outpatient department, exhibited relatively high resistance patterns towards Trimethoprim, Sulfamethoxazole and Nalidixic acid at range of $60 \%$ to 80\% (Table 8).

Table 7. Resistance profiles of Gram-positive isolated bacteria pathogens.

\begin{tabular}{|c|c|c|c|c|c|c|c|c|c|c|c|c|c|c|c|c|c|c|c|c|c|c|c|}
\hline Organism & Comment & $n$ & AMP & $\mathrm{CAZ}$ & CRO & CTX & ATM & FEP & AMC & AMK & GEN & CIP & NAL & $\mathrm{CHL}$ & ERY & FOX & LNZ & NIT & RIF & SMX & $\mathrm{TCY}$ & TMP & VAN \\
\hline Enterococus & UTI & 1 & 0 & 0 & 0 & 0 & 0 & 0 & 0 & 0 & 0 & 0 & 100 & 0 & 100 & 0 & 100 & 0 & 100 & 100 & 0 & 100 & 100 \\
\hline spp. & Contaminants & 4 & 50 & 25 & 50 & 50 & 33 & 25 & 50 & 67 & 50 & 0 & 75 & 25 & 67 & 50 & 33 & 25 & 67 & 75 & 50 & 50 & 33 \\
\hline$S$ & UTI & 5 & 60 & 40 & 40 & 40 & 40 & 40 & 40 & 0 & 0 & 0 & 80 & 20 & 40 & 40 & 40 & 20 & 60 & 60 & 80 & 80 & 0 \\
\hline saprophyticus & Contaminants & 3 & 33 & 33 & 33 & 33 & 33 & 33 & 33 & 0 & 0 & 0 & 100 & 0 & 33 & 33 & 33 & 0 & 33 & 67 & 0 & 67 & 0 \\
\hline & UTI & 12 & 33 & 17 & 25 & 25 & 56 & 17 & 17 & 11 & 0 & 0 & 67 & 8 & 57 & 18 & 30 & 18 & 22 & 67 & 25 & 75 & 0 \\
\hline & Contaminants & 7 & 29 & 29 & 29 & 29 & 33 & 29 & 29 & 33 & 29 & 0 & 100 & 14 & 50 & 29 & 50 & 0 & 50 & 71 & 57 & 57 & 0 \\
\hline
\end{tabular}

Key: Antimicrobial susceptibility patterns of both significant and non-significant (contaminants) isolates from the study participants. The ranges to determine where the isolates were sensitive (S), intermediate (I) or resistance (R) were done as documented before [33]. Zero \% ( $0 \%$ ) means no resistance noted. Antibiotics routinely used in UTI management of both gram-positive and gram-negative bacteria: Ampicillin (AMP), Amoxicillin-clavulanic acid(AMC), Ceftriaxone (CRO), Nitrofurantoin (NIT), Gentamin (GEN), Cefoxitin (FOX), Erythromycin (ERY), Ciprofloxacin (CIP), Cefepime (FEP), Rifampicin (RIF), Nalidixic acid (NAL), Ceftazidime (CAZ), Cefotaxime (CTX), Sulfamethoxazole (SMX), Trimethoprim (TMP), Chloramphenicol (CHL), Tetracycline (TCY), Linezolid (LNZ), Vancomycin (VAN), Aztreonam (ATM).

Table 8. Resistance profiles of isolated bacteria pathogens based on department category.

\begin{tabular}{|c|c|c|c|c|c|c|c|c|c|c|c|c|c|c|c|c|c|c|c|c|c|c|c|}
\hline Organism & $\begin{array}{c}\text { Admission } \\
\text { type }\end{array}$ & $n$ & AMC & AMK & AMP & ATM & CAZ & $\mathrm{CHL}$ & CIP & CRO & CTX & ERY & FEP & FOX & GEN & LNZ & NAL & NIT & RIF & SMX & TCY & TMP & VAN \\
\hline \multirow[t]{2}{*}{ E. coli } & Inpatient & 3 & 33 & 0 & 33 & 0 & 33 & 0 & 33 & 33 & 33 & 0 & 33 & 50 & 33 & 0 & 67 & 67 & 0 & 33 & 0 & 67 & 0 \\
\hline & Outpatient & 26 & 15 & 28 & 19 & 42 & 15 & 12 & 26 & 19 & 19 & 71 & 15 & 19 & 26 & 28 & 42 & 19 & 43 & 58 & 39 & 42 & 0 \\
\hline Enterococcus sp. & Outpatient & 5 & 0 & 0 & 20 & 0 & 0 & 0 & 0 & 0 & 0 & 0 & 0 & 0 & 0 & 0 & 80 & 0 & 0 & 80 & 40 & 60 & 67 \\
\hline Klebsiella sp. & Outpatient & 11 & 9 & 0 & 18 & 20 & 0 & 0 & 9 & 18 & 18 & 40 & 0 & 9 & 9 & 20 & 73 & 18 & 0 & 73 & 27 & 91 & 0 \\
\hline \multirow[t]{2}{*}{ Proteus sp. } & Inpatient & 1 & 0 & 0 & 0 & 0 & 0 & 0 & 0 & 0 & 0 & 0 & 0 & 0 & 0 & 0 & 100 & 0 & 0 & 0 & 0 & 0 & 0 \\
\hline & Outpatient & 8 & 38 & 0 & 50 & 0 & 25 & 25 & 38 & 50 & 50 & 100 & 13 & 13 & 0 & 100 & 88 & 25 & 100 & 50 & 50 & 75 & 0 \\
\hline S. saprophyticus & Outpatient & 8 & 38 & 0 & 75 & 50 & 25 & 25 & 29 & 25 & 38 & 50 & 25 & 25 & 25 & 50 & 75 & 14 & 50 & 75 & 25 & 63 & 0 \\
\hline S. aureus & Outpatient & 17 & 41 & 29 & 88 & 43 & 18 & 13 & 35 & 24 & 24 & 71 & 18 & 14 & 18 & 71 & 65 & 12 & 86 & 59 & 59 & 50 & 0 \\
\hline
\end{tabular}

Key: Antimicrobial susceptibility patterns of isolates from the study participants. The ranges to determine where the isolates were sensitive (S), intermediate (I) or resistance (R) were done as documented before [33]. Zero \% (0\%) means no resistance noted. Antibiotics routinely used in UTI management of both Gram-positive and Gram-negative bacteria: Ampicillin (AMP), Amoxicillin-clavulanic acid(AMC), Ceftriaxone (CRO), Nitrofurantoin (NIT), Gentamin (GEN), Cefoxitin (FOX), Erythromycin (ERY), Ciprofloxacin (CIP), Cefepime (FEP), Rifampicin (RIF), Nalidixic acid (NAL), Ceftazidime (CAZ), Cefotaxime (CTX), Sulfamethoxazole (SMX), Trimethoprim (TMP), Chloramphenicol (CHL), Tetracycline (TCY), Linezolid (LNZ), Vancomycin (VAN), Aztreonam (ATM). 


\subsubsection{Resistance Profiles of Isolated Bacterial Pathogens Based on Gender}

Resistance patterns based on gender revealed that isolates obtained from the female gender exhibited drug and multi-drug resistance to most of the study drugs. For instance, the resistance patterns noted among $E$. coli isolates from the female cohort against Ampicillin, Ciprofloxacin and Nitrofurantoin were at a range of $20 \%$ to $40 \%$. The obtained $E$. coli isolates among the male gender were all sensitive towards Amoxicillin-clavulanic acid, Ceftriaxone and Cefepime a complete opposite to E. coli isolates from the female gender that showed $20 \%$, $24 \%$ and $20 \%$ resistance patterns respectively (Table 9 ).

\section{Discussion}

Urinary tract infections continue to be more prevalent in the hospital setting globally. In Africa, for instance, a study conducted at Okada in Nigeria reported an overall prevalence rate of $39.69 \%$ [15]. However, in this study the overall UTI prevalence rate was $27.6 \%$, which is still high although falls within the global range of $13 \%-33 \%$ [22], an almost similar UTI prevalence rate was also reported in Ismailia City, at $29 \%$ [2]. Even so, a more UTI prevalence variation of $90.1 \%$ was noted in a study conducted at Shashemene referral hospital, Ethiopia [15] an indication that these infections vary globally. Regionally at Mulago Hospital, Uganda, a UTI prevalence rate of $13.3 \%$ was reported [17]. Much of the same prevalence findings of $24 \%$, was reported at the Aga Khan University Hospital, Nairobi, Kenya [18] and going by these aforementioned regional prevalence rates, there is a positive sign that implies that locally urinary tract infection management policies are moderately adhered to but the literature to enlighten the public domain is still limited. This study data analysis disclosed that majority

Table 9. Resistance profiles of isolated bacterial pathogens based on gender.

\begin{tabular}{|c|c|c|c|c|c|c|c|c|c|c|c|c|c|c|c|c|c|c|c|c|c|c|c|}
\hline Organism & Sex & $\mathbf{N}$ & AMC & AMK & AMP & ATM & $\mathrm{CAZ}$ & $\mathrm{CHL}$ & CIP & CRO & CTX & ERY & FEP & FOX & GEN & LNZ & NAL & NIT & RIF & SMX & TCY & TMP & VAN \\
\hline Escherichia coli & Female & 25 & 20 & 29 & 24 & 43 & 20 & 13 & 28 & 24 & 24 & 71 & 20 & 24 & 28 & 29 & 40 & 28 & 43 & 56 & 36 & 40 & 0 \\
\hline Enterococcus sp. & Female & 5 & 0 & 0 & 20 & 0 & 0 & 0 & 0 & 0 & 0 & 0 & 0 & 0 & 0 & 0 & 80 & 0 & 0 & 80 & 40 & 60 & 67 \\
\hline \multirow[t]{2}{*}{ Klebsiella sp. } & Female & 15 & 7 & 20 & 20 & 40 & 0 & 0 & 7 & 13 & 13 & 20 & 0 & 7 & 7 & 20 & 67 & 7 & 20 & 60 & 27 & 80 & 0 \\
\hline & Male & 1 & 0 & 0 & 0 & 0 & 0 & 0 & 0 & 0 & 0 & 100 & 0 & 0 & 0 & 100 & 0 & 100 & 0 & 100 & 0 & 100 & 0 \\
\hline Proteus sp. & Female & 9 & 33 & 0 & 44 & 0 & 22 & 22 & 33 & 44 & 44 & 100 & 11 & 11 & 0 & 100 & 89 & 22 & 100 & 44 & 44 & 67 & 0 \\
\hline S. saprophyticus & Female & 8 & 38 & 0 & 75 & 50 & 25 & 25 & 29 & 25 & 38 & 50 & 25 & 25 & 25 & 50 & 75 & 14 & 50 & 75 & 25 & 63 & 0 \\
\hline S. aureus & Female & 18 & 39 & 29 & 89 & 43 & 17 & 18 & 33 & 22 & 22 & 71 & 17 & 13 & 19 & 71 & 68 & 11 & 86 & 56 & 50 & 47 & 0 \\
\hline
\end{tabular}

Key: Antimicrobial susceptibility patterns of isolates from the study participants. The ranges to determine where the isolates were sensitive ( $S$ ), intermediate (I) or resistance (R) were done as documented before [33]. Zero \% (0\%) means no resistance noted. Antibiotics routinely used in UTI management of both Gram-positive and Gram-negative bacteria: Ampicillin (AMP), Amoxicillin-clavulanic acid (AMC), Ceftriaxone (CRO), Nitrofurantoin (NIT), Gentamin (GEN), Cefoxitin (FOX), Erythromycin (ERY), Ciprofloxacin (CIP), Cefepime (FEP), Rifampicin (RIF), Nalidixic acid (NAL), Ceftazidime (CAZ), Cefotaxime (CTX), Sulfamethoxazole (SMX), Trimethoprim (TMP), Chloramphenicol (CHL), Tetracycline (TCY), Linezolid (LNZ), Vancomycin (VAN), Aztreonam (ATM). 
of UTIs cases were still among women at $80.7 \%$ compared to men $19.2 \%$. The finding supports that females continue to be more vulnerable to contracting UTI due to their basic anatomy, whereby their urethra is closer to the anal opening and shorter than men's [8] [10] [11] [22]. Again, women's hormonal fluctuations across the menstrual cycle and the thought of a genetic factor that tends to run in families may also be fuelling their vulnerability [8] [20] [22] [27].

Further considerations of the reported urinary tract infection prevalence rates among the different participants' cohorts suggested that UTI occurrences may be influenced by the various social demographic characteristics and life style factors that came into place (Tables 2-5). Past related studies have also reported different factors that seem to heighten UTI contraction. Amongst such factors included; individuals changing their social habits, age, social economic status and health conditions [1]. In this study the possible linkage and associated risk factors that partially seemed to fuel contraction of UTI were investigated. How informed the study participants were about urinary tract infection was also considered, with a significantly low number of participants (14\%) having no idea. Based on the study socio-demographic characteristics data, the prevalence rate of urinary tract infection in relation to age was at $29.2 \%$ among those aged between 21 - 30 years. This was low compared to that among those aged between 41 - 50 years of $42.9 \%$. However, those aged above 50 years had a UTI prevalence rate of $27.3 \%$, which was afflicted to most of them having other underlying co-morbidities like diabetic and indwelling catheters (Table 2). The UTI prevalence among those over 50 years did concur to the finding reported in Nigeria by Anuli et al. 2016 where most of the elderly participants' also had an increased risk of developing UTI due to factors such as kidney stones, hormonal changes or prostrate problems [1].

Sexual activities, wearing non-cotton underwear and poor personal hygiene have previously been documented to be key factors that influence contraction of UTI [18]. Much of the same findings were revealed by the data analysis reports in relation to frequent sexual activity, wearing non-cotton underpants, and poor personal hygiene (Table 2). Study participants within the cohort of $\leq 30$ years had UTI positivity rate of $31 \%$ and agreed to be sexually active more than twice a week. This finding is in tandem and supported the views documented in prior research studies on how during sexual intercourse, thrusting could introduce bacteria up the urethra and into the bladder, increasing the risk of UTI contraction [12] [28]. Again, the finding on the poor personal hygiene also seconds that such conditions create a suitable environment for pathogens to thrive (Table 4). Therefore there is the need to enlighten individuals on optimum personal hygiene to halt UTI contraction. In addition, knowing the individual's knowledge and population-specific UTI-associated risk factors may help tailor prophylactic strategies to curb contraction UTIs [37].

The outpatient department shows antenatal participants recording the highest UTI prevalence rate of (34\%). This is in agreement with the finding of a study by 
Dimetry et al. 2007 where high UTI prevalence rate was reported among pregnant women [38]. Again, the reported urinary tract infection prevalence among pregnant women in this study compared to that of $29 \%$ reported at Ismailia city, Egypt [2] and that of $15.7 \%$ reported at Pumwani, Kenya [25] is higher and signals a worrying health concern that needs a prudent solution. More clinical and laboratory investigations to determine the factors that may be by far be influencing UTI occurrence in pregnancy must be embraced. Furthermore, the American pregnancy association did record that the uterus sits on top of the bladder, and during pregnancy as the uterus grows; the increase in weight blocks the drainage of the urine from the bladder causing an infection. In addition, the high sugar and protein levels in the urine of pregnant mothers may fuel the contraction of UTI [8] [20] [22]. However, according to the study data analysis there was no significant association of UTI contraction with demographic characteristics such as: marital status, level of education, occupation, social economic status, and blood pressure readings (Table 2 and Table 5).

Quite a number of bacterial etiological agents were isolated as responsible for UTI among the study population (Table 1). This finding is not unique to this study and is in tandem to other previous UTI studies [11] [15] [18] [25]. E. coli was the commonest isolated bacteria pathogen at (38.5\%), followed by Staphylococcus aureus (21\%) and Klebsiella pneumonia (19.3\%) respectively. Analysis of the isolate's data provided crucial information which revealed drug and multi-drug resistance patterns amongst both Gram-positive and Gram-negative bacteria (Table 6 and Table 7). The noted resistance patterns may be attributed to various captured factors amongst the participants. These included purchasing of antibiotics over the counter without proper prescription that leads to drug misuse and promotes antimicrobial resistance trends since there is no relevant laboratory investigation to reveal drug susceptibility. Again, study participants admitted to the use of antibiotics as a prophylactic measure, and delay to seek medical attention on time, a situation that may be fuelling treatment failure. Considering all these aforementioned factors and the revealed resistance findings endorses that antimicrobial resistance is on an exponential stage. E. coli and Staphylococcus aureus specifically exhibited the highest resistance trends at a range of $33 \%$ to $75 \%$ towards Ampicillin, Tetracycline and Sulfamethoxazole (Table 6 and Table 7). These findings concur with other related studies from the regional that documented E. coli being resistant to most of the studies test antibiotics [7] [18] [39].

The susceptibility profile of Staphylococcus aureus against Ceftriaxone was at $25 \%$, similar to that noted at Gondar Teaching Hospital, Ethiopia [22] but different to that of 37\% reported at Agha Khan University Hospital [18]. This is an encouraging observation that indicates that with prudent antimicrobial stewardship, antimicrobial resistance trends can be halted. However, going by the resistance patterns of Klebsiella pneumoniae isolates of $27 \%$ compared to that of $E$. coli at $14 \%$ against Nitrofurantoin there is still a huge gap that re- 
quires to be addressed. Again, the demonstrated resistance trends against Sulfurmethoxazole and Nalidixic acid by most of the study isolates between $60 \%$ to $80 \%$ does concur with the findings by Masika et al. 2017 [39]. It is therefore plausible that pathogens from this region may have developed other survival mechanisms to enable their resistance development to commonly used antibiotics over the years.

The noticed antimicrobial resistance (AMR) and multiple drug resistance (MDR) findings can also be attributed to long-term empirical prescription of most of the study drugs over the years. For instance, going by the noted resistance trends, Sulfurmethoxazole and Ampicillin are no longer potent enough to be prescribed as 1st line antibiotics in empirical management. Therefore, due to this increased AMR and MDR, more alternative antibiotic breakthroughs are awaited with many optimizations worldwide. Campaigning for the enlightenment of the public domain to discourage the use of antibiotics in a prophylactic measure rather than a therapeutic measure will be very essential to retain efficacy of the drugs that remain. However, there is also the need to periodically revise the country's UTI treatment guidelines to ensure that some antibiotics are reserved only for the very critical established infections. Holistic scientific research to unearth uropathogens genotypic carriage of ESBLs resistance genes that may be mediating resistance against antibiotics is also indispensable [40]. This will assist in obtaining phylogeny data that will shed light on the genetic relatedness of bacterial isolates and explain whether any major clones are circulating in the population, fueling treatment failure. Additionally, crucial information on whether uropathogens have developed traits that may negatively impact antibiotics' cidal actions will be achieved. The developments of vaccines and drug sensitivity rapid diagnostic kits as future prospect are also firmly recommended since this will help minimise the turnaround time for culture microbiology results, hamper UTI contraction, and minimise the overreliance of empirical UTI management.

\section{Conclusion}

According to our findings, UTI is still a very common infection among adults requiring efficient, prompt diagnosis and treatment. The unearthed study UTI prevalence rate certainly justifies the need for further investigations to improve on diagnosis, management and shun recurrence. Again, the finding of scrutinized risk associated factors plainly endorses the need to regularly explain the circumstances that may be by degrees fuelling the contraction of urinary tract infection. Also, it is distinct that management of UTIs without relevant microbial data is prone to inappropriate antibiotic prescription hence the need to embrace urine culture in every UTI management to promote proper antimicrobial stewardship. Furthermore, this will offer support to pharmaceutical industries that have fewer new antibiotics in the pipeline. However, due to the revealed study isolates resistance trends, a long-lasting solution to stagnate uropathogens rising antibiotic resistance is urgent. 


\section{Acknowledgements}

To the study participants, thank you for agreeing to take part in this study. The authors wish to further express heartfelt gratitude to all KEMRI/CMR (molecular biology) staff members for their invaluable assistance. We are also grateful to the clinicians, laboratory technologists, and the administration Kiambu level 5 Hospital for their guidance and support, which allowed this study to succeed. This study was funded by HATUA (Holistic Approach to Unravel Antimicrobial resistance in East Africa) research funds. The funders had no role in study design, data collection and analysis, decision to publish, or manuscript preparation.

\section{Authors' Contribution}

Fredrick Wanja (FW) was the principal investigator and came up with the study concept with guidance from Dr. Eric Omwenga. FW designed, carried out the lab work, data analysis and drafted the manuscript. John Maina participated in sample collection and drafting of the manuscript. Dr. Caroline Ngugi and Dr. Eric Omwenga assisted in preparation and harmonization of the manuscript. Dr. John Kiiru took part in the study design and supervised the lab bench work. All authors read and endorsed the manuscript.

\section{Authors' Information}

Fredrick Kimunya (FK) is a master's student in Kenya studying Infectious Diseases and Vaccinology at Jomo Kenyatta University of agriculture and technology. John Maina (JM) is a scientific researcher at the Centre of Microbiology Research, Kenya Medical Research Institute, Nairobi, Kenya. Dr. Caroline Ngugi (CN) is the Chairperson Department of Medical Microbiology, Jomo Kenyatta University of Agriculture and Technology who possesses vast experience in proposal development. Dr. Eric Omwenga (EO) is the Chairperson Department of Microbiology \& Parasitological, Kisii University. He assisted in coming up with the study concept and revision of the content of the manuscript. Dr. John Kiiru (JK) took part in the study design and actualization of the content of the manuscript. He is a senior researcher at the Centre of Microbiology Research, Kenya Medical Research Institute, Nairobi, Kenya and the Head of Laboratory Ministry of Health, Nairobi, Kenya who has vast knowledge in infectious diseases and microbiology.

\section{Ethical Approval}

Approved (Supplementary Data S1 and Supplementary Data S2).

\section{Conflicts of Interest}

The authors declare no conflicts of interest regarding the publication of this paper. 


\section{References}

[1] John, A.S., Mboto, C.I. and Agbo, B. (2016) A Review on the Prevalence and Predisposing Factors Responsible for Urinary Tract Infection among Adults. European Journal of Experimental Biology, 6, 7-11.

[2] Mohamed, N.R., Omar, H.H.H. and Abd-Allah, I.M. (2017) Prevalence and Risk Factors of Urinary Tract Infection among Pregnant Women in Ismailia City, Egypt. IOSR Journal of Nursing and Health Science, 6, 62-72.

[3] Ciani, O., Grassi, D. and Tarricone, R. (2013) An Economic Perspective on Urinary Tract Infection: The "Costs of Resignation". Clinical Drug Investigation, 33, 255-261. https://doi.org/10.1007/s40261-013-0069-X

[4] Mohiuddin, A.K. (2019) Alternative Management of Uncomplicated UTIs in Women. Journal of Gynecology and Women's Health, 16, Article ID: 555930. https://doi.org/10.19080/JGWH.2019.14.555930

[5] Leone, M., Garnier, F., Avidan, M. and Martin, C. (2004) Catheter-Associated Urinary Tract Infections in Intensive Care Units. Microbes and Infection, 6, 1026-1032. https://doi.org/10.1016/j.micinf.2004.05.016

[6] Tasota, F.J., Fisher, E.M., Coulson, C.F. and Hoffman, L.A. (1998) Protecting ICU Patients from Nosocomial Infections: Practical Measures for Favorable Outcomes. Critical Care Nurse, 18, 54-65. https://doi.org/10.4037/ccn1998.18.1.54

[7] Odoki, M., Almustapha Aliero, A., Tibyangye, J., Nyabayo Maniga, J., Wampande, E., Drago Kato, C., et al. (2019) Prevalence of bacterial Urinary Tract Infections and Associated Factors among Patients Attending Hospitals in Bushenyi District, Uganda. International Journal of Microbiology, 2019, Article ID: 4246780.

https://doi.org/10.1155/2019/4246780

[8] Al-Badr, A. and Al-Shaikh, G. (2013) Recurrent Urinary Tract Infections Management in Women: A Review. Sultan Qaboos University Medical Journal, 13, 359-367.

[9] Lema, V.M. (2015) Urinary Tract Infection in Young Healthy Women Following Heterosexual Anal Intercourse. African Journal of Reproductive Health, 19, 134-139.

[10] Scholes, D., Hooton, T.M., Roberts, P.L, Stapleton, A.E., Gupta, K. and Stamm, W.E. (2020) Risk Factors for Recurrent Urinary Tract Infection in Young Women. The Journal of Infectious Diseases, 182, 1177-1182. https://doi.org/10.1086/315827

[11] Tan, C. and Chlebicki, M. (2016) Urinary Tract Infections in Adults. Singapore Medical Journal, 57, 485-490. https://doi.org/10.11622/smedj.2016153

[12] Vasudevan, R. (2014) Urinary Tract Infection: An Overview of the Infection and the Associated Risk Factors. Journal of Microbiology \& Experimentation, 1, 42-54. https://doi.org/10.15406/jmen.2014.01.00008

[13] Sammon, J.D., Sharma, P., Rahbar, H., Roghmann, F., Ghani, K.R., Sukumar, S., et al. (2014) Predictors of Admission in Patients Presenting to the Emergency Department with Urinary Tract Infection. World Journal of Urology, 32, 813-819. https://doi.org/10.1007/s00345-013-1167-3

[14] He, K., Hu, Y., Shi, J.C., Zhu, Y.Q. and Mao, X.M. (2018) Prevalence, Risk Factors and Microorganisms of Urinary Tract Infections in Patients with Type 2 Diabetes Mellitus: A Retrospective Study in China. Therapeutics and Clinical Risk Management, 14, 403-408. https://doi.org/10.2147/TCRM.S147078

[15] Oladeinde, B., Omoregie, R., Mitsan, O. and Anunibe, J. (2011) Urinary Tract Infection in a Rural Community of Nigeria North. North American Journal of Medical Sciences, 3, 75-77.

[16] Seifu, W. and Gebissa, A. (2018) Prevalence and Antibiotic Susceptibility of Uropa- 
thogens from Cases of Urinary Tract Infections (UTI) in Shashemene Referral Hospital, Ethiopia. BMC Infectious Diseases, 18, Article No. 30. https://doi.org/10.1186/s12879-017-2911-X

[17] Alemu, A., Moges, F., Shiferaw, Y., Tafess, K., Kassu, A., Anagaw, B., et al. (2012) Bacterial Profile and Drug Susceptibility Pattern of Urinary Tract Infection in Pregnant Women at University of Gondar Teaching Hospital, Northwest Ethiopia. BMC Research Notes, 5, Article No. 197. https://doi.org/10.1186/1756-0500-5-197

[18] Kabugo, D., Kizito, S., Ashok, D.D., Kiwanuka, A.G., Nabimba, R., Namunana, S., et al. (2016) Factors Associated with Community-Acquired Urinary Tract Infections among Adults Attending Assessment Centre, Mulago Hospital Uganda. African Health Sciences, 16, 1131-1142. https://doi.org/10.4314/ahs.v16i4.31

[19] Okinda, N. and Revathi, G. (2012) Urinary Tract Infections at Aga Khan University Hospital Nairobi-A One Year Experience. East African Medical Journal, 89, 147-153.

[20] Sharifian, M., Karimi, A., Rafiei Tabatabaei, S. and Anvaripour, N. (2007) Microbial Sensitivity Pattern in Urinary Tract Infection in Children: A Single Center Experience of 1,177 Urine Cultures. Japanese Journal of Infectious Diseases, 59, 380-382.

[21] Watson, A. (2007) Management of Urinary Tract Infection in Children. BMJ, 335, 356-357. https://doi.org/10.1136/bmj.39309.423542.80

[22] Ikram, R., Psutka, R., Carter A. and Priest, P. (2015) An Outbreak of Multi-Drug Resistant Escherichia coli Urinary Tract Infection in an Elderly Population: A Case-Control Study of Risk Factors. BMC Infectious Diseases, 15, Article No. 224. https://doi.org/10.1186/s12879-015-0974-0

[23] Foxman, B. (2002) Epidemiology of Urinary Tract Infections: Incidence, Morbidity, and Economic Costs. The American Journal of Medicine, 113, 5-13. https://doi.org/10.1016/S0002-9343(02)01054-9

[24] Bau, G.M. (2015) Deciphering the Early Events Leading to an Adaptive Immune Response during Urinary Tract Infection. PhD Thesis, Université Pierre et Marie Curie, Paris.

[25] Onyango, H.A., Ngugi, C., Maina, J. and Kiiru, J. (2018) Urinary Tract Infection among Pregnant Women at Pumwani Maternity Hospital, Nairobi, Kenya: Bacterial Etiologic Agents, Antimicrobial Susceptibility Profiles and Associated Risk Factors. Advances in Microbiology, 8, 175-187. https://doi.org/10.4236/aim.2018.83012

[26] Nicolle, L.E. (2003) Asymptomatic Bacteriuria: When to Screen and When to Treat. Infectious Disease Clinics, 17, 367-394.

https://doi.org/10.1016/S0891-5520(03)00008-4

[27] Rowe, T.A. and Juthani-Mehta, M. (2014) Diagnosis and Management of Urinary Tract Infection in Older Adults. Infectious Disease Clinics of North America, 28, 75-89. https://doi.org/10.1016/j.idc.2013.10.004

[28] Gerda, A. and Sleep, D.A.D. (2011) Urinary Tract Infection (UTI).

[29] Tena, D., Gonzílez-Praetorius, A., Síez-Nieto, J.A., Valdezate, S. and Bisquert, J. (2008) Urinary Tract Infection Caused by Capnophilic Escherichia coli. Emerging Infectious Diseases, 14, 1163-1164. https://doi.org/10.3201/eid1407.071053

[30] Gezmu, T., Regassa, B., Manilal, A., Mama, M. and Merdekios, B. (2016) Prevalence, Diversity and Antimicrobial Resistance of Bacteria Isolated from the UTI Patients of Arba Minch Province, Southern Ethiopia. Translational Biomedicine, 7, Article No. 3.

[31] Monica, C. (2000) Biochemical Tests to Identify Bacteria. In District Laboratory Practice in Tropical Countries, Cambridge University Press, Cambridge, New York, 
UK, 62-70.

[32] World Health Organization (2003) Manual for the Laboratory Identification and Antimicrobial Susceptibility Testing of Bacterial Pathogens of Public Health Importance in the Developing World: Haemophilus influenzae, Neisseria meningitidis, Streptococcus pneumoniae, Neisseria gonorrhoea, Salmonella serotype Typhi, Shigella, and Vibrio cholerae. World Health Organization, Geneva.

[33] Bauer, A.W. (1966) Antibiotic Susceptibility Testing by a Standardized Single Disc Method. American Journal of Clinical Pathology, 45, 493-496. https://doi.org/10.1093/ajcp/45.4 ts.493

[34] Clinical and Laboratory Standards Institute (CLSI) (2009) Performance Standards for Antimicrobial Disk Diffusion Susceptibility Tests. 19th Edition Approved Standard. Clinical and Laboratory Standards Institute, Wayne, CLSI Document M100-S19, 29, M100-MS21.

[35] Vercauteren, E., Descheemaeker, P., Ieven, M., Sanders, C.C. and Goossens, H. (1997) Comparison of Screening Methods for Detection of Extended-Spectrum Beta-Lactamases and Their Prevalence among Blood Isolates of Escherichia coli and Klebsiella spp. in a Belgian Teaching Hospital. Journal of Clinical Microbiology, 35, 2191-2197. https://doi.org/10.1128/jcm.35.9.2191-2197.1997

[36] Bedenic, B., Randegger, C., Boras, A. and Haechler H. (2001) Comparison of Five Different Methods for Detection of SHV Extended-Spectrum $\beta$-Lactamases. Journal of Chemotherapy, 13, 24-33. https://doi.org/10.1179/joc.2001.13.1.24

[37] Storme, O., Tiran Saucedo, J., Garcia-Mora, A., Dehesa-Dívila, M. and Naber, K.G. (2019) Risk Factors and Predisposing Conditions for Urinary Tract Infection. Therapeutic Advances in Urology, 11, 1756287218814382. https://doi.org/10.1177\%2F1756287218814382

[38] Dimetry, S.R., El-Tokhy, H.M., Abdo, N.M., Ebrahim, M.A. and Eissa, M. (2007) Urinary Tract Infection and Adverse Outcome of Pregnancy. The Journal of the Egyptian Public Health Association, 82, 203-218.

[39] Masika, W.G., O’Meara, W.P., Holland, T.L. and Armstrong, J. (2017) Contribution of Urinary Tract Infection to the Burden of Febrile Illnesses in Young Children in Rural Kenya. PLoS ONE, 12, e0174199. https://doi.org/10.1371/journal.pone.0174199

[40] Lim, Y.K., Lee, M.K. and Kim, T.H. (2015) Management of Extended-Spectrum Beta-Lactamase-Positive Gram-Negative Bacterial Urologic Infections. Urogenital Tract Infection, 10, Article No. 8. https://doi.org/10.14777/uti.2015.10.2.84 


\section{Supplementary Data S1}

\section{Ethical approval from the $\mathrm{KNH}-\mathrm{U}$ oN ethical research committee}

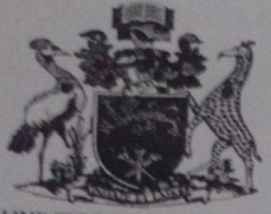

UNIVERSITY OF NAIROBI

COLLEGE OF HEALTH SCIENCES

PO BOX 19676 Code 00202

Telegrams: varsity

Tel:(254-020) 2726300 Ext 44355

\section{Ref: $\mathrm{KNH}-\mathrm{ERC} / \mathrm{A} / 470$}

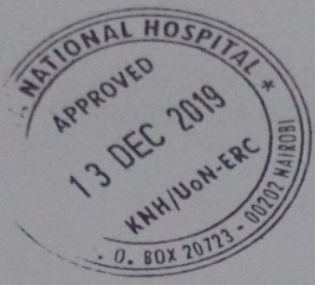

KNH-UON ERC

Email: uonknh_erc@uonbi.ac.ke

Website: http://www.erc.uonbi.ac.ke Facebook: https://www.facebook.com/uonknh.erc Twitter: @UONKNH_ERC https://twitter.com/UONKNH_ERC

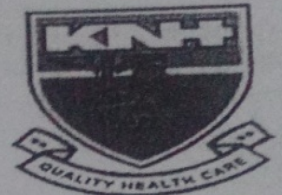

KENYATTA NATIONAL HOSPITAL

P O BOX 20723 Code 00202

Tel: $726300-9$

Fax: 725272

Telegrams: MEDSUP, Nairobi

$13^{\text {th }}$ December, 2019

Fredrick Kimunya Wanja

Reg. No.HSB-325-1358/17

Dept. of Medical Microbiology

College of Health Sciences (CoHES)

J.KU.A.T

\section{Dear Fredrick}

RESEARCH PROPOSAL: PREVALENCE, ANTIMICROBIAL SUSCEPTIBILITY PROFILES AND GENOTYPIC CHARACTERIZATION OF BACTERIAL ISOLATES OBTAINED FROM URINE SAMPLES OF ADULT PATIENTS ATTENDING KIAMBU LEVEL 5 HOSPITAL, KENYA (P691/08/2019)

This is to inform you that the KNH- UoN Ethics \& Research Committee (KNH- UoN ERC) has reviewed and approved your above research proposal. The approval period is $13^{\text {th }}$ December $2019-12^{\text {th }}$ December 2020.

This approval is subject to compliance with the following requirements:

a. Only approved documents (informed consents, study instruments, advertising materials etc) will be used.

b. All changes (amendments, deviations, violations etc.) are submitted for review and approval by KNH-UoN ERC before implementation.

c. Death and life threatening problems and serious adverse events (SAEs) or unexpected adverse events whether related or unrelated to the study must be reported to the KNH-UoN ERC within 72 hours of notification.

d. Any changes, anticipated or otherwise that may increase the risks or affect safety or welfare of study participants and others or affect the integrity of the research must be reported to KNH- UoN ERC within 72 hours.

e. Clearance for export of biological specimens must be obtained from $\mathrm{KNH}$ - UoN ERC for each batch of shipment.

f. Submission of a request for renewal of approval at least 60 days prior to expiry of the approval period. (Attach a comprehensive progress report to support the renewal).

g. Submission of an executive summary report within 90 days upon completion of the study. This information will form part of the data base that will be consulted in future when processing related research studies so as to minimize chances of study duplication and/ or plagiarism. 


\section{Supplementary Data S2}

\section{Ethical approval from National Commission for Science, Technology and Innovation (NACOSTI)}

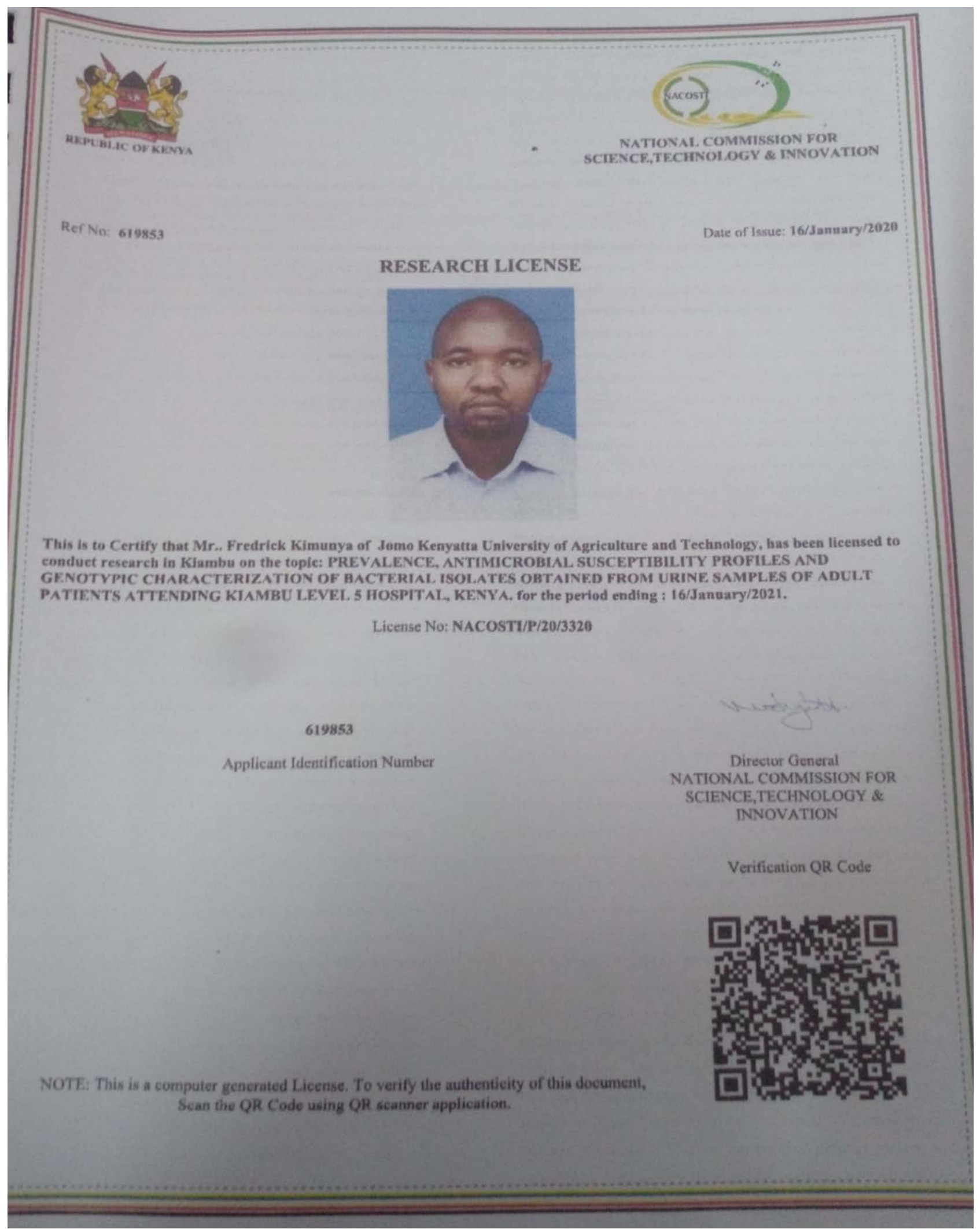




\section{Supplementary Data S3}

\section{Examples of study isolates' colony morphologies}

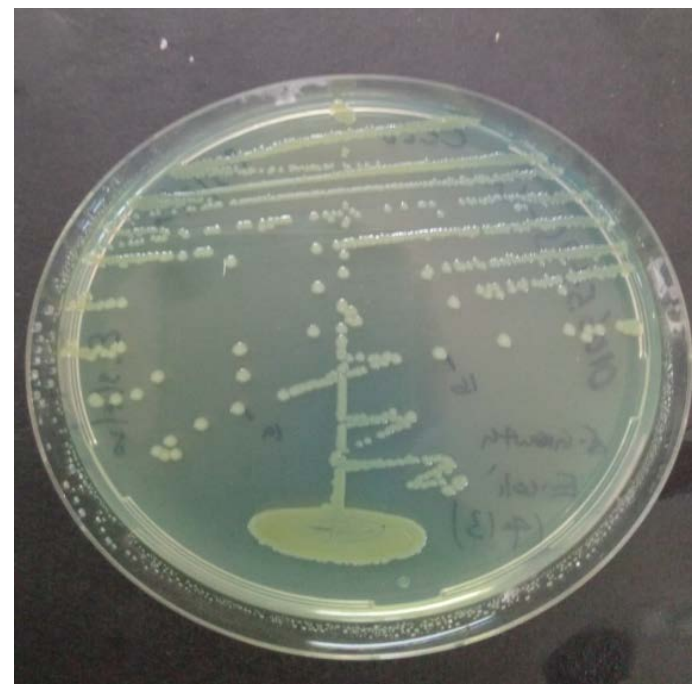

(a) Gram negative (Escherichia coli)

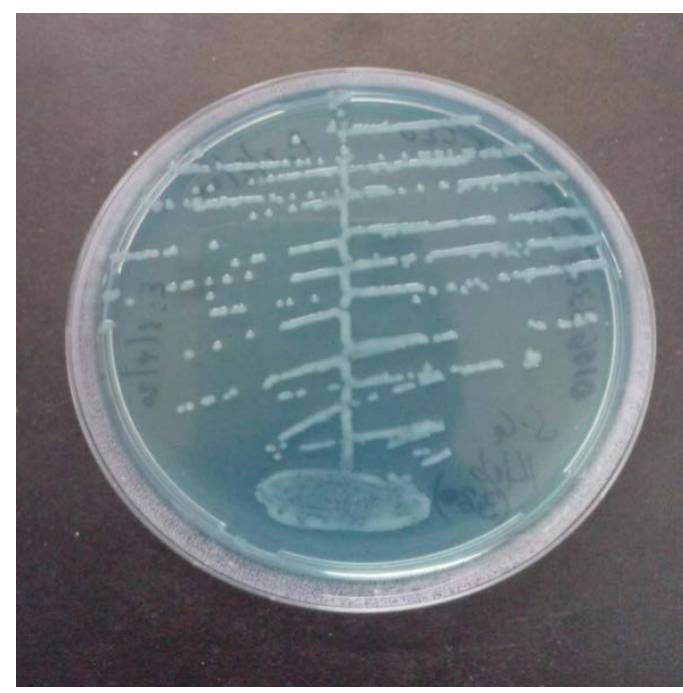

(b) Gram-negative (Proteus mirabilis)

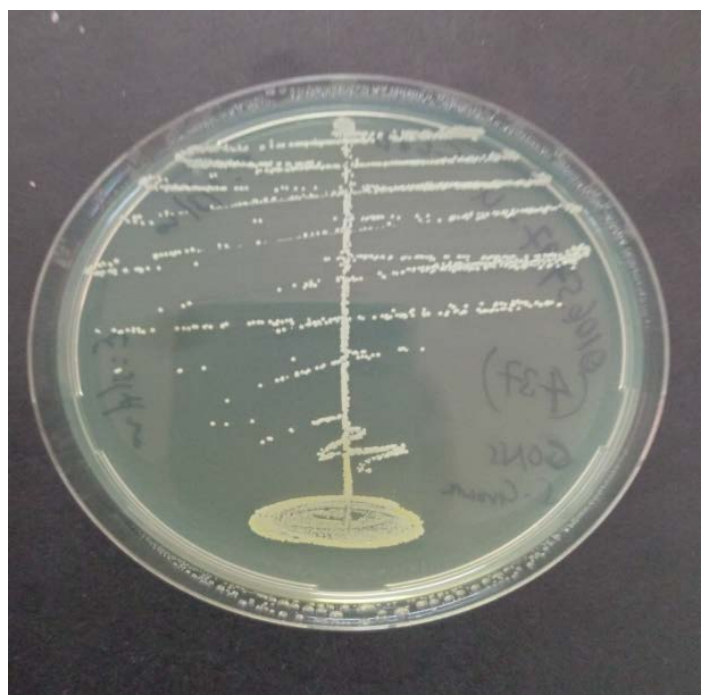

(c) Gram-positive (Staphylococcus aureus) 